\title{
Thalidomide enhances cyclophosphamide and dexamethasone- mediated cytotoxicity towards cultured chronic lymphocytic leukaemia cells
}

\author{
JOANNA C. POINTON ${ }^{1,2}$, GINA EAGLE ${ }^{2}$, JAMES BAILEY ${ }^{2}$, PAUL EVANS ${ }^{4}$, \\ DAVID ALLSUP $^{3}$ and JOHN GREENMAN ${ }^{1,2}$ \\ ${ }^{1}$ Centre for Biomedical Research, University of Hull, Cottingham Road, Hull, HU6 7RX; ${ }^{2}$ Daisy Clinical Research Facility \\ and ${ }^{3}$ Department of Haematology, Castle Hill Hospital, Castle Road, Cottingham, East Yorkshire, HU16 5JQ, UK; \\ ${ }^{4}$ HMDS Laboratory, Leeds Teaching Hospital NHS Trust, Leeds, LS9 7TF, UK
}

Received June 4, 2010; Accepted July 2, 2010

DOI: 10.3892/or_00000988

\begin{abstract}
Numerous chemotherapeutic regimens exist for the treatment of symptomatic or progressive chronic lymphocytic leukaemia (CLL). However, once the disease becomes refractory to nucleoside-based therapy the prognosis is poor. In this study we investigated the cytotoxicity of thalidomide in combination with dexamethasone, fludarabine and cyclophosphamide. Cells from a cohort of 25 CLL patients were incubated for $72 \mathrm{~h}$ with each of these three agents, at 3 concentrations, both with and without thalidomide. Cell viability was assessed using the Annexin V:FITC assay. Fludarabine was highly toxic to the cells, producing very high levels of cell death; however, thalidomide did not increase this effect. Cyclophosphamide combined with thalidomide showed a small, non-significant improvement in toxicity compared with monotherapy. Median cell death for $5 \mu \mathrm{M}$ dexamethasone monotherapy and for combination with thalidomide was $15 \%$ [interquartile range (IQR) 0-38\%] and 17\% (IQR 0-54\%), respectively (Wilcoxon Signed Rank analysis, $\mathrm{p}=0.034$ ). Cell death for $10 \mu \mathrm{M}$ dexamethasone monotherapy was $15 \%$ (IQR $0-45 \%$ ) and $16 \%$ (IQR 0-62\%) in combination with thalidomide (Wilcoxon Signed Rank analysis, $\mathrm{p}=0.035$ ). At the highest doses tested 11 of 25 cases displayed an enhancement of cyclophosphamide-mediated cytotoxicity, and 14 of 25 cases showed enhanced dexamethasone-mediated cytotoxicity in the presence of thalidomide. Some CLL cells in which dexamethasone-mediated killing was enhanced were derived from patients with poor prognostic markers, including p53 mutations and unmutated $\operatorname{IgV}_{\mathrm{H}}$ genes. In summary, thalidomide enhances cyclophosphamide- and dexamethasone-
\end{abstract}

Correspondence to: Professor John Greenman, Centre for Biomedical Research, University of Hull, Cottingham Road, Hull, HU6 7RX, UK

E-mail: j.greenman@hull.ac.uk

Key words: CLL, thalidomide, dexamethasone, apoptosis, chemotherapy mediated cytotoxicity of CLL cells in vitro in a proportion of cases.

\section{Introduction}

Chronic lymphocytic leukaemia (CLL) is the most commonly occurring leukaemia in the Western world, is more prevalent in men than women, and has a median age of diagnosis of 65 years (1-3). The disease is characterised by a proliferation and accumulation of small, homogeneous, mature B-lymphocytes in the blood, bone marrow, lymph nodes and spleen. In contrast to many malignancies, CLL proliferates at a relatively slow rate with the cells escaping the fate of programmed cell death (PCD or apoptosis) $(4,5)$.

CLL is incurable, but symptomatic disease can be effectively treated with purine analogues, glucocorticoids, alkylating agents, monoclonal antibodies or combinations of these (6). The overall response and clinical outcome when using any therapeutic agent depends on several factors, including previous treatment, clinical stage and cytogenetic abnormalities making it very difficult to find a successful treatment for every patient (7). Patients with relapsed or refractory disease have limited treatment options, as few therapies are effective (8). Poor prognostic factors include unmutated immunoglobulin heavy chain variable regions $\left(\mathrm{IgV}_{\mathrm{H}}\right)$, expression of $\mathrm{CD} 38$ and zeta-associated protein 70 (ZAP-70) (1).

Chlorambucil or fludarabine are usually incorporated into initial treatment regimens but rates of complete remission with these drugs as monotherapy is low. Cyclophosphamide has been evaluated extensively within combination regimens for CLL with both the cyclophosphamide, pentostatin, rituximab and fludarabine, cyclophosphamide, rituximab regimens producing excellent response rates $(9,10)$. CLL cells display in vitro sensitivity to the cytotoxic effects of dexamethasone (6) and this agent has been incorporated into chemotherapy regimens found to be effective in the treatment of this disease $(11,12)$.

Thalidomide is an immunomodulating agent that has been found to be an anti-angiogenic, antiproliferative and proapoptotic agent which acts through the inhibition of "pro- 
survival' cytokines such as tumour necrosis factor $\alpha$ (TNF- $\alpha$ ), interleukin-12 and other pro-inflammatory Th1 type cytokines $(7,13,14)$. Thalidomide has been intensively investigated in multiple myeloma (MM), both as a monotherapy and in combination with other agents. Rajkumar and colleagues showed that thalidomide in combination with dexamethasone yielded superior response rates compared with dexamethasone, however, a greater toxicity was seen with the combination therapy (15). Fludarabine has also been assessed in combination with thalidomide for the treatment of CLL; a phase I clinical trial demonstrated an overall response rate of $100 \%$, with $55 \%$ achieving a complete remission (13).

The mechanism(s) of action of thalidomide are still being investigated, as are the possible treatment options utilising this drug. Here we have assessed the synergistic effect of combining thalidomide with fludarabine, cyclophosphamide and dexamethasone in vitro, looking at cell survival to discover new drug combinations with clinical efficacy in CLL.

\section{Materials and methods}

Chronic lymphocytic leukaemia patients. The study utilised peripheral blood samples taken from 25 patients with typical CLL (mature lymphocytes expressing CD5, CD19, CD23 and clonally restricted surface immunoglobulin), all of whom possessed a white blood cell count $\geq 20 \times 10^{9} / 1$. The study had Local Research Ethics Committee (Ref. 05/Q1104/33) and NHS Hull and East Yorkshire Trust approval. Informed consent was obtained from all patients prior to recruitment. Mononuclear cells were isolated from samples by centrifugation on a Ficoll-Hypaque gradient, using Histopaque ${ }^{{ }_{-}}$ 1077 (Sigma), according to manufacturer's protocol, and cryopreserved in liquid nitrogen in the presence of $10 \%(\mathrm{v} / \mathrm{v})$ dimethyl sulphoxide (DMSO). Before use, the cells were thawed at $37^{\circ} \mathrm{C}$ and the DMSO diluted to $0.5 \%(\mathrm{v} / \mathrm{v})$ in media over a period of $20 \mathrm{~min}$. Mean viability of the CLL-cells on thawing as assessed by trypan blue exclusion was $80.4 \%$.

The patient cohort (Table I) comprised 13 males and 12 females, with a mean age of 72 years (range $37-87$ ). The majority of patients were classed as early stage disease, either Rai 0 or Binet A (64 and 76\%, respectively); however, a number of patients showed signs of poor risk markers such as the expression of ZAP-70 in $10 \%$ or greater of the malignant cells $\left(23 \%\right.$ of cases), unmutated $\mathrm{IgV}_{\mathrm{H}}$ genes $(26 \%)$, deletion of $17 \mathrm{p}$ or $11 \mathrm{q}(28 \%)$ and the expression of CD38 by $30 \%$ or more of the CLL-cells (24\%). The average number of previous treatment cycles was 1.5 (range $0-5$ ).

Reagents and media. Fludarabine (2-fluoroadenine-9-ß-Darabinofuranoside), cyclophosphamide monohydrate and dexamethasone crystalline were obtained from Sigma Chemical Co., Ltd. (Poole, UK); thalidomide was provided by Pharmion Corp. (Berkshire, UK). Roswell Park Memorial Institute (RPMI) 1640 culture medium with $300 \mu \mathrm{g} / \mathrm{ml} \mathrm{L}$ glutamine (PAA; The Cell Culture Co., Somerset, UK) supplemented with $1 \%(\mathrm{w} / \mathrm{v})$ bovine serum albumin (BSA; Fisher Scientific, Loughborough, UK) and penicillinstreptomycin solution (final concentration $100 \mathrm{U} / \mathrm{ml}$ penicillin and $100 \mu \mathrm{g} / \mathrm{ml}$ streptomycin; Sigma). This medium was sterilized by filtering through a $0.2-\mu \mathrm{m}$ syringe filter (Anachem, Bedfordshire, UK).
Table I. Summary of clinical characteristics for the patient cohort.

\begin{tabular}{lr} 
Characteristics & No. of pa \\
\hline Gender & \\
$\quad$ Male & 13 \\
Female & 12 \\
Age (years), mean (range) & $72(37-86$ \\
Binet stage (at diagnosis) & \\
A & 19 \\
B & 5 \\
C & 1
\end{tabular}

Rai stage (at diagnosis)

0

I

II

III

IV

\section{FISH}

Isolated deletion $13 \mathrm{q}$

Trisomy 12

Deletion $11 \mathrm{q}$

Deletion $17 \mathrm{p}$

Normal 2

Not tested

$\operatorname{IgV} \mathrm{V}_{\mathrm{H}}$ mutation status

Mutated (<98\%)

Unmutated (>98\%)

Not tested

ZAP-70 (by flow cytometry)

Positive 5

Negative $\quad 17$

Not tested 3

CD38 expression

Positive 4

Negative 13

Not tested 8

No. of previous therapies, mean (range) $1.5(0-5)$

Culture of cells. Cells were incubated at $37^{\circ} \mathrm{C}$ in a humidified atmosphere containing $5 \% \mathrm{CO}_{2}$. Cells were incubated at a concentration of $1 \times 10^{6}$ cells/ml for $72 \mathrm{~h}$ with 9,18 or $36 \mu \mathrm{M}$ cyclophosphamide, $2.5,5$ or $10 \mu \mathrm{M}$ dexamethasone, $1,2.2$ or $4.4 \mu \mathrm{M}$ fludarabine, $400 \mu \mathrm{M}$ thalidomide or a combination of agents, as above, with thalidomide. Hydrogen peroxide (2.9 M) was used as a positive control for inducing cell death, by incubating an aliquot of cells for the final $30 \mathrm{~min}$ of the 72-h incubation period with $100 \mu 1$ of this solution. 
Analysis of cell viability by Annexin $V$ binding. Cell survival was assessed using the Annexin V:FITC assay kit (AbD Serotec, Oxford, UK). Briefly, following incubation, cells were washed in ice-cold phosphate-buffered saline (PBS; $0.01 \mathrm{M}$ phosphate buffer, $2.7 \mu \mathrm{M}$ potassium chloride, $137 \mu \mathrm{M}$ sodium chloride, pH 7.4; Sigma) resuspended in $200 \mu 1$ binding buffer, incubated with $5 \mu \mathrm{l}$ Annexin $\mathrm{V}$ :fluorescein isothiocyanate (FITC) for $10 \mathrm{~min}$ in the dark; and finally washed again in PBS. Cells were then resuspended in $200 \mu 1$ binding buffer and incubated with $10 \mu 1$ propidium iodide (PI) before sample analysis on a FACSCalibur flow cytometer (Becton-Dickinson, Oxford, UK). Ten thousand cells per sample were acquired and analysed using CellQuest software (Version 3.3; BectonDickinson). All experiments were done in duplicate.

Cell survival was investigated by dividing the flow cytometric data into viable, necrotic and apoptotic populations. Comparisons of the percentage of dead cells (apoptotic and apoptotic plus necrotic) demonstrated any effects of treatment with cyclophosphamide, dexamethasone or fludarabine either alone or in combination with thalidomide. The percentage of cell death given by the untreated cells incubated in similar conditions was defined as the baseline; hence all test conditions were normalised against this condition to allow comparison.

Analysis of $\operatorname{Ig} V_{H}$ mutational status, fluorescent in situ hybridisation, ZAP-70 expression and proportion of CD19 positive cells. RNA for the $\mathrm{IgV}_{\mathrm{H}}$ analysis was extracted using the Qiagen RNeasy mini kit (Qiagen, Crawley, UK) and the Reverse-iT kit (ABgene, Epsom, UK) was used to synthesise complementary DNA. The $\operatorname{IgV}_{\mathrm{H}}$ gene was amplified using PCR, as previously described (16), and the BIG dye terminator sequencing kit version 3 (Applied Biosystems, Warrington, UK) was used for sequencing. Sequences were compared with germline using IgBLAST (National Centre for Biotechnology Information, Bethesda, Maryland; http://ncbi.nih. gov/igblast/). Those with homology of $\geq 98 \%$ were classified as unmutated and those with $<98 \%$ homology were classified as mutated (17).

Fluorescent in situ hybridisation for deletions in $13 \mathrm{q}, 11 \mathrm{q}$ and $17 \mathrm{p}$ as well as for trisomy 12 were performed according to published methodologies (18).

ZAP-70 and CD19 expression were assessed by flow cytometry with reference to an isotypic control (19). A threshold of $10 \%$ ZAP-70 positive cells was used to differentiate ZAP-70 positivity and negativity. Following a viable cell count, $4 \times 10^{5}$ cells were incubated with mouse anti-human CD19:RPE (AbD Serotec, Oxford, UK) for $30 \mathrm{~min}$, after which cells were then incubated with $100 \mu$ l fixation reagent (Reagent A, Leucoperm; AbD Serotec). Following washing with PBS permeabilisation reagent (100 $\mu$ l Reagent B, Leucoperm) was added to the cells along with the mouse anti-human ZAP-70 Alexa Fluor 488 (Caltag Laboratories, Paisley, UK). The flow cytometer was set to acquire 10,000 events for each sample and data were analysed as described above.

Statistical analysis. Distribution of data was analysed using tests for skewness and kurtosis. All data were found to be non-normally distributed. The significance of differences was therefore evaluated by the Wilcoxon Signed Rank test at $\mathrm{p}<0.05$ level of significance. All statistics were performed using the SPSS 15.0 software package (SPSS Inc.).

\section{Results}

To assess the effects of the cytotoxic drugs with and without thalidomide, it was necessary to maintain the cells in culture for $72 \mathrm{~h}$. CLL cells do not survive well in vitro, thus it was necessary to identify a cohort of patients with CLL cells whose survival was $\geq 30 \%$ after $72 \mathrm{~h}$ that could be used for the cytotoxicity assays. Forty-five samples were analysed (median survival following $72 \mathrm{~h}$ culture was 31\% (IQR 9-50\%; range $1-79 \%$ ) and it was only possible to utilise 25 samples for subsequent studies. This cohort was subsequently tested to assess the B-cell population using CD19 by flow cytometry; the median B-cell population was $96 \%$ (IQR 92-97\%).

Thalidomide monotherapy. Dose ranging experiments with thalidomide were performed on a total of 10 independent patient samples using concentrations of $0.1-1 \mathrm{mM}$. Thalidomide showed some, limited effects on CLL cell viability when used as monotherapy, and a concentration of $400 \mu \mathrm{M}$ was selected for use in all subsequent experiments. This value represents a maximal level achievable in plasma levels in vivo. At this concentration cells from 12 of the 25 cases tested demonstrated an increase in cell death relative to untreated CLL-cells following $72 \mathrm{~h}$ incubation with 3 of these cases demonstrating a $>20 \%$ increase in cell death relative to untreated cells. An incubation period of $72 \mathrm{~h}$ was chosen as time course experiments demonstrated little increase in thalidomide induced CLL-cell death following culture for 24-48 h.

Fludarabine therapy in combination with thalidomide. Median cell death for fludarabine alone at the lowest dose tested, $1 \mu \mathrm{M}$ was $71 \%$ (IQR 48-92\%) and in combination with thalidomide was $69 \%$ (IQR 35-91\%). At $4.4 \mu \mathrm{M}$ the highest concentration of fludarabine used, cell death was 94\% (IQR 86-98\%) and in combination with thalidomide was 91\% (IQR 70-96\%). Fludarabine alone produced extremely high levels of cell death, which the addition of thalidomide did not further enhance.

Cyclophosphamide therapy in combination with thalidomide. Median cell death for cyclophosphamide at $9 \mu \mathrm{M}$, the lowest concentration tested, was $2 \%$ (IQR $0-7 \%$ ) and $1 \%$ (IQR 0$18 \%$ ) when used in combination with thalidomide. At $18 \mu \mathrm{M}$, median cell death with cyclophosphamide was 6\% (IQR 0$13 \%$ ) and 5\% (IQR 0-22\%) when combined with thalidomide. Finally at the highest concentration, $36 \mu \mathrm{M}$ cell death was 9\% (IQR 2-22\%) and 10\% (IQR 0-25\%) alone and combined with thalidomide, respectively. Of the 25 samples tested, thalidomide increased the proportion of dead cells in 8 cases when co-incubated with cyclophosphamide at $9 \mu \mathrm{M}, 12$ cases when co-incubated with cyclophosphamide $18 \mu \mathrm{M}$ and 11 cases when co-incubated with cyclophosphamide at $36 \mu \mathrm{M}$. Fig. 1 displays the data on the cytotoxic effects of thalidomide and cyclophosphamide monotherapy, and the combination of these two agents towards cultured CLL cells using the 


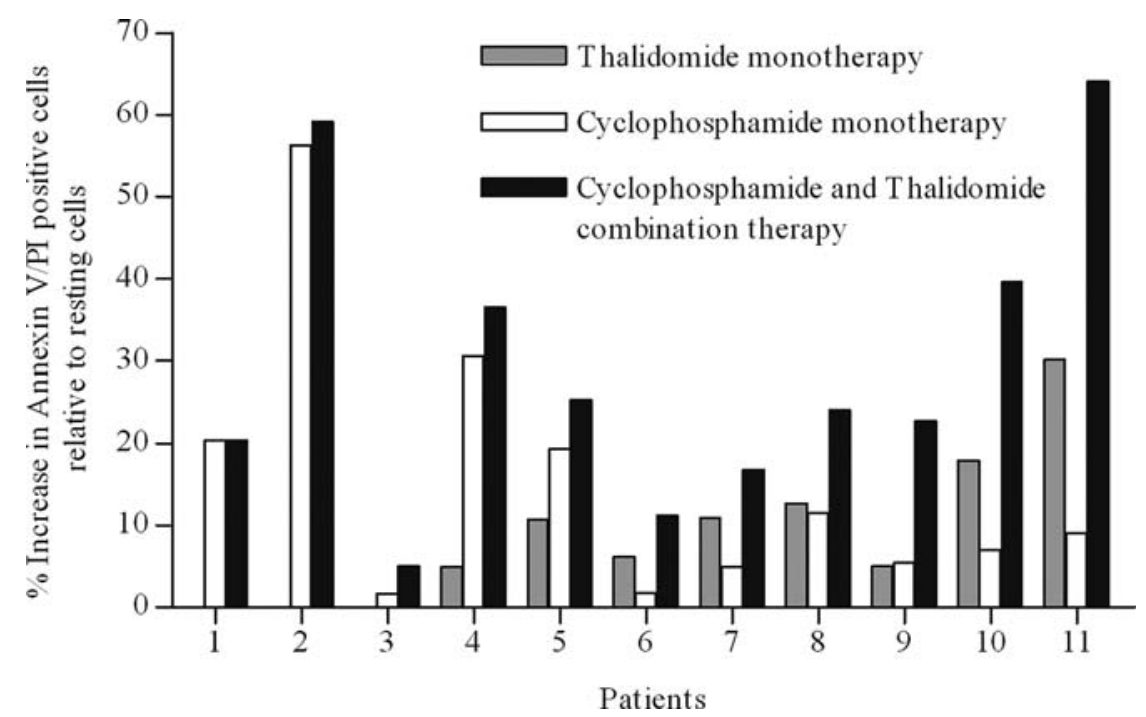

Figure 1. The percentage of dead, Annexin V/PI positive CLL cells from individual patient samples when treated with thalidomide (400 $\mu \mathrm{M})$, cyclophosphamide $(36 \mu \mathrm{M})$ or a combination of both are shown following incubation for $72 \mathrm{~h}$. Cell death in response to cytotoxics is expressed as a percentage increase relative to the number of dead cells noted in the absence of exposure to cytotoxics under identical culure conditions. Results are arranged in order of increasing difference between treatment with cyclophosphamide alone and in combination with thalidomide. In patients 1-3 thalidomide alone caused no cell death. Only the 11 patients that showed an increase in cytotoxicity are displayed.
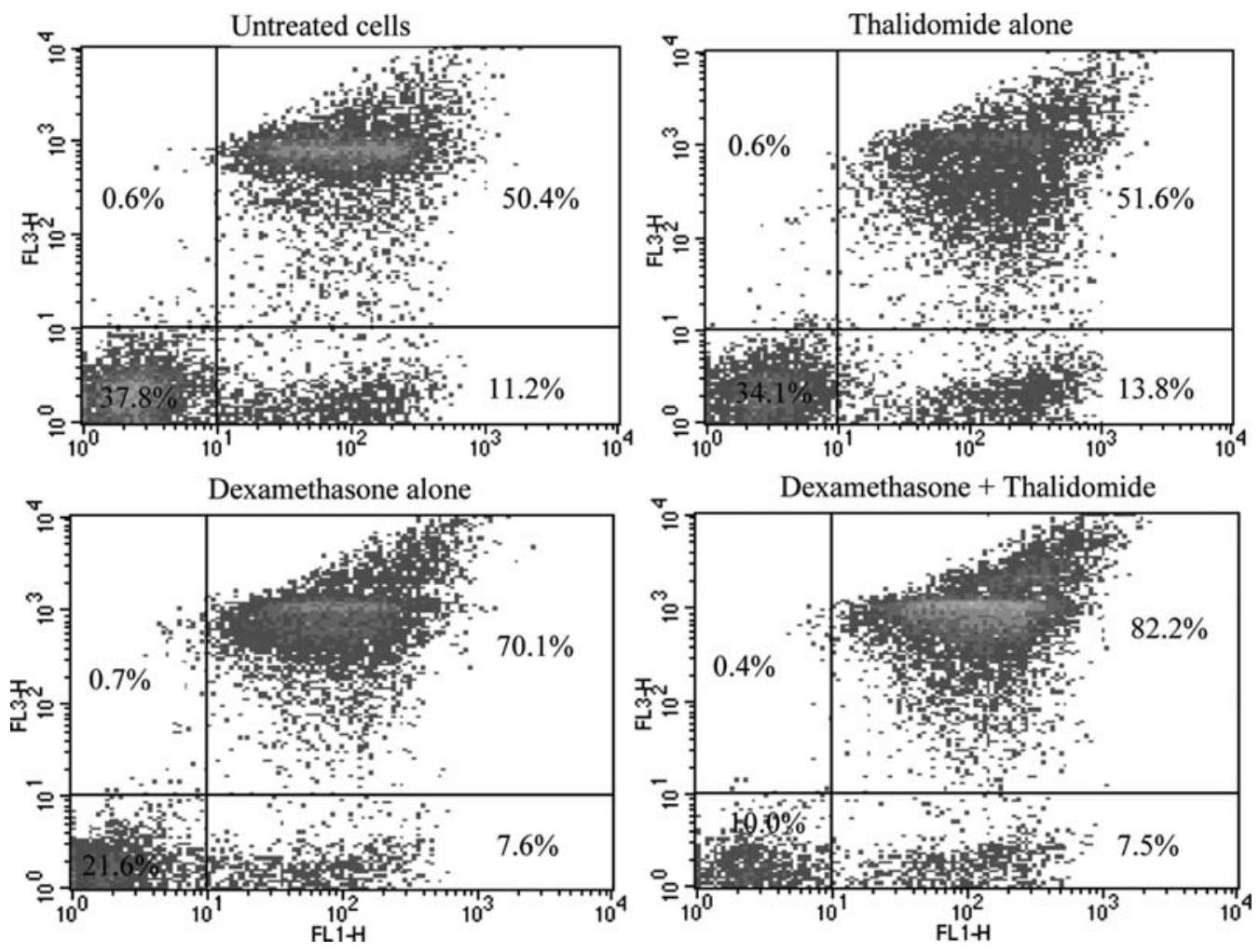

Figure 2. FACS plots of CLL-cells incubated for $72 \mathrm{~h}$ in the presence of media, thalidomide $400 \mu \mathrm{M}$, dexamethasone $10 \mu \mathrm{M}$ or thalidomide $400 \mu \mathrm{M}$ and dexamethasone $10 \mu \mathrm{M}$. The x-axis (FL-1) indicates Annexin V expression whilst the y-axis (FL-3) represents PI uptake. Events within the lower left quadrant of each plot represent Annexin $\mathrm{V}^{-} / \mathrm{PI}^{-}$viable cells whilst events within the upper right quadrant of each plot represent Annexin $\mathrm{V}^{+} / \mathrm{PI}^{+}$dead cells. Overall cell death was taken to be the total of Annexin $\mathrm{V}^{+} / \mathrm{PI}^{-}$, Annexin $\mathrm{V}^{+} / \mathrm{PI}^{+}$and Annexin $\mathrm{V}^{-} / \mathrm{PI}^{+}$cells. Percentages correspond to the quadrant where the figure is located. A total of 10,000 events were counted for each plot.

highest concentration of cyclophosphamide tested, in the 11 cases that showed an enhancement of the cytotoxic effect of cyclophosphamide by thalidomide.
Of the 11 cases in which thalidomide enhanced cyclophosphamide-mediated cytotoxicity towards CLL cells, in four cases the malignant cells expressed unmutated $\operatorname{IgV}_{\mathrm{H}}$ 


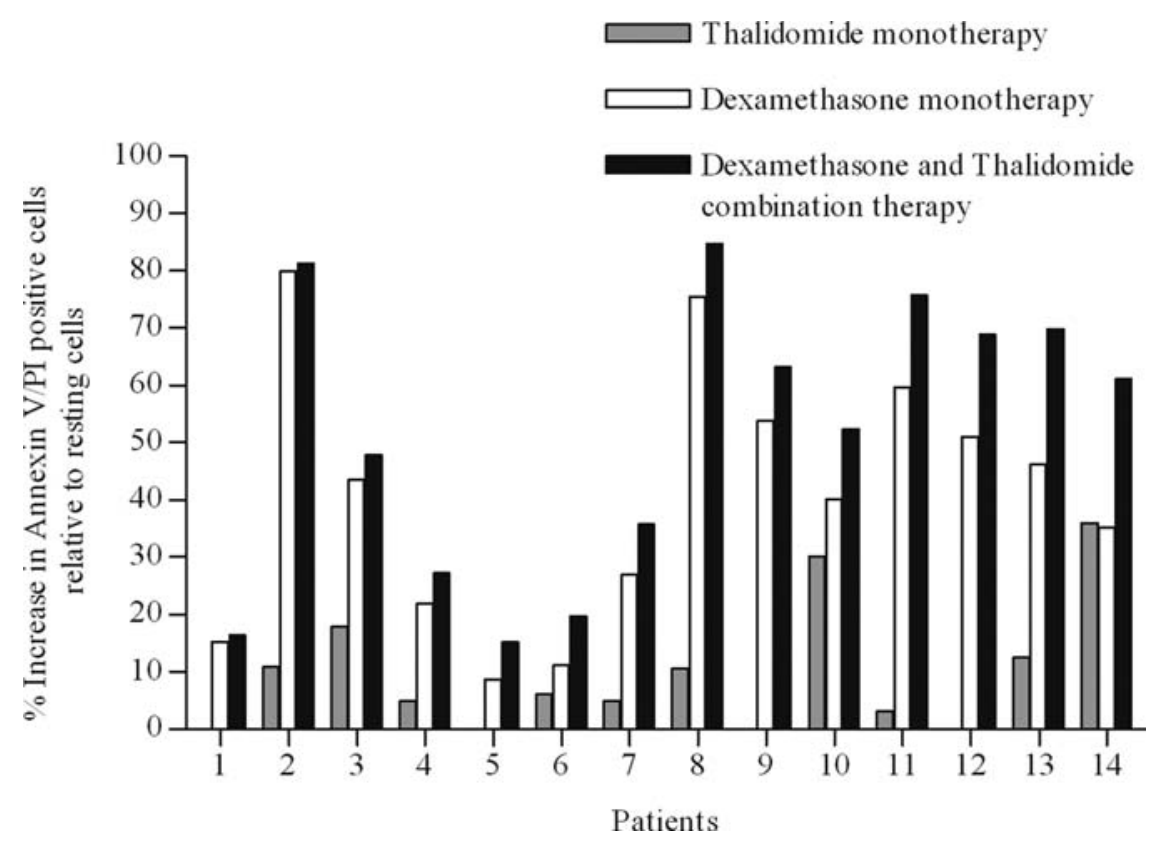

Figure 3. The percentage of dead, Annexin V/PI positive CLL cells from individual patient samples when treated with thalidomide (400 $\mu \mathrm{M}$ ), dexamethasone $(10 \mu \mathrm{M})$ or a combination of both are shown following incubation for $72 \mathrm{~h}$. Cell death in response to cytotoxics is expressed as a percentage increase relative to the number of dead cells noted in the absence of exposure to cytotoxics under identical culure conditions. The results are arranged in order of increasing difference between treatment with dexamethasone alone and in combination with thalidomide. In patients $1,5,9$ and 12 thalidomide alone caused no cell death. Only the 14 patients that showed an increase in cytotoxicity are displayed.

genes (data available for 10), three were ZAP-70 positive, one was CD38 positive (data available for 8 ) and two had a deletion $17 \mathrm{p}$ (data available for 14). The patients from which these samples were taken had received a mean of 1.4 previous courses of therapy (range $=0-3$ ).

Dexamethasone therapy in combination with thalidomide. Thalidomide enhanced the cytotoxic effect of dexamethasone against CLL cells in 11 of 24 cases when co-incubated with $2.5 \mu \mathrm{M}$ dexamethasone, in 10 of 25 cases when co-incubated with $5 \mu \mathrm{M}$ dexamethasone, and in 14 of 25 cases when coincubated with $10 \mu \mathrm{M}$ dexamethasone. Median cell death for dexamethasone at $2.5 \mu \mathrm{M}$ was $12 \%$ (IQR $0-42 \%$ ) and $14 \%$ (IQR 0-51\%) when in combination with thalidomide ( $\mathrm{p}=0.121)$. In the presence of $5 \mu \mathrm{M}$ dexamethasone median cell death was $15 \%$ (IQR $0-38 \%$ ) and $17 \%$ (IQR $0-54 \%$ ) on coincubation with thalidomide $(\mathrm{p}=0.034)$. At the highest concentration $(10 \mu \mathrm{M})$ median cell death with dexamethasone as a single agent was $15 \%$ (IQR $0-45 \%$ ) and $16 \%$ (IQR 0-62\%) on co-incubation with thalidomide ( $\mathrm{p}=0.035$; Figs. 2 and 3 ). Therefore significantly elevated levels of cell death were observed using a combination of 5 and $10 \mu \mathrm{M}$ dexamethasone and thalidomide, as compared with dexamethasone alone.

Of the fourteen cases in which thalidomide enhanced dexamethasone $(10 \mu \mathrm{M})$ mediated cytotoxicity towards CLL cells, the malignant cells from four cases expressed unmutated $\mathrm{IgV}_{\mathrm{H}}$ genes (data available for 13), four were ZAP-70 positive (data available for 13), three were CD38 positive (data available for 11) and three possessed a deletion $17 \mathrm{p}$ (data available for 14). The patients from which these samples were taken had received a mean of 1 previous course of therapy (range 0-3).

\section{Discussion}

Fludarabine-based chemotherapy regimens are considered to be effective in the treatment of CLL, with a superior overall response compared with other treatment regimens, with overall response rates of $75-80 \%$ (20). However, some patients become refractory to fludarabine and form a unique group associated with shortened survival and a marked susceptibility to infections $(21,22)$.

A phase I clinical trial was carried out to establish the safety and efficacy of combining fludarabine with thalidomide in previously untreated CLL patients. This yielded encouraging results, with an overall response rate of $100 \%$ and a complete remission rate of $55 \%$ (13). In an attempt to understand the mechanism by which thalidomide enhances fludarabine mediated cytotoxicity, Laurenti and colleagues measured the levels of TNF- $\alpha$ in five, heavily pre-treated, patients, undergoing combination therapy with cyclophosphamide, fludarabine and thalidomide (23). TNF- $\alpha$ is known to cause tumour proliferation of some malignancies, and thalidomide has previously been shown to inhibit production of this cytokine (24). However, serum TNF- $\alpha$ levels in these patient did not show any sign of decrease during treatment, but this may be due, at least in part, to the drug resistance caused by several previous rounds of chemotherapy and the fact that all the patients had to stop treatment early due to disease progression or neurological toxicity.

Previously Podhorecka et al have conducted an in vitro study to assess the interactions of thalidomide with fludarabine in terms of induction of apoptosis (25). At early time-points $(<24 \mathrm{~h})$ thalidomide induced DNA damage as evidenced by increased phosphorylation of histone $\mathrm{H} 2 \mathrm{AX}$ as well as inducing low levels of the apoptotic marker caspase-3, however thalido- 
mide did not increase overall CLL-cell death when used as monotherapy. Thalidomide and fludarabine in combination demonstrated an enhancement of fludarabine induced CLLcell killing suggesting that thalidomide may 'prime' CLLcells for nucleoside-induced cytotoxicity by co-inducing lowlevels of DNA damage. Our study in contrast demonstrated that following prolonged CLL-cell incubation $(72 \mathrm{~h})$ with thalidomide a minority of cases of CLL displayed increased cell death but that fludarabine-induced cell death was maximal and not further enhanced by thalidomide. This suggests that in vitro thalidomide may initially sensitise CLL-cells to fludarabine-mediated cytotoxicity resulting in more rapid cell killing. In addition thalidomide has been noted to reduce levels of regulatory T-cells in vivo possibly facilitating a direct cytotoxic T-cell effect against CLL-cells (26).

In the present study it was found that cyclophosphamide was relatively ineffective both with and without thalidomide. Eleven of the 25 samples showed an increase in cell death when treated with cyclophosphamide and thalidomide in comparison to cyclophosphamide alone. Eight of these samples had a difference between the induced cell death of $\geq 5 \%$. As expected, dexamethasone displayed significant cytotoxic activity against cultured CLL cells, and 14 of the 25 samples tested showed an increase in cell death when treated with combined dexamethasone and thalidomide, in comparison with dexamethasone alone. Eleven of these samples showed a difference between the induced cell death of $\geq 5 \%$ including some from patients with adverse prognostic factors.

Corticosteroids exert their cytotoxic effect in a p53independent manner and the observation that two of the patients in whom thalidomide enhanced dexamethasone mediated-cytotoxicity towards cultured CLL cells possessed a p53 deletion suggests that the effect of thalidomide on CLL cell viability may also be independent of p53 activity. CLL patients whose malignant cells possess p53 deletions have a very poor prognosis, with a median survival of less than 18 months, and show a weak response to alkylators and purine analogues. Combinations of agents such as alemtuzumab and methylprednisolone, whose cytotoxic activity is independent of p53, have been investigated therapeutically in CLL with some success although at the expense of considerable toxicity (27). Our results suggest that a thalidomide/corticosteroid-based regimen may be effective in a proportion of CLL patients, including those with poor prognostic features.

The combination of thalidomide with either cyclophosphamide or dexamethasone is associated with a modest enhancement of the in vitro cytotoxicity of these latter two agents towards CLL cells. However, as thalidomide has complex anti-angiogenic and anti-inflammatory effects when used in vivo for the treatment of malignancy it is likely that the relatively small increase in cytotoxic effect observed in vitro, will be further enhanced in a clinical setting. Thalidomide and dexamethasone may therefore be a rational combination on which to base the development of future chemotherapy regimens for the treatment of CLL.

\section{Acknowledgements}

We would like to thank the Haematological Malignancy Diagnostic Service, Leeds General Infirmary, Leeds, for providing $\operatorname{IgV}_{\mathrm{H}}$ mutation and CD38 expression data, and Hull York Medical School and Pharmion Corp. for their financial support. These data were presented at the British Society of Haematology conference, Glasgow, April, 2008.

\section{References}

1. Ghia P, Ferreri AM and Caligaris-Cappio F: Chronic lymphocytic leukemia. Crit Rev Oncol Hematol 64: 234-246, 2007.

2. Jemal A, Siegel R, Ward E, Murray T, Xu J and Thun MJ: Cancer statistics, 2007. CA Cancer J Clin 57: 43-66, 2007.

3. Rozman C and Montserrat E: Chronic lymphocytic leukemia. N Engl J Med 333: 1052-1057, 1995.

4. Danilov AV, Danilova OV, Klein AK and Huber BT: Molecular pathogenesis of chronic lymphocytic leukemia. Curr Mol Med 6: 665-675, 2006.

5. Meinhardt G, Wendtner CM and Hallek M: Molecular pathogenesis of chronic lymphocytic leukemia: factors and signaling pathways regulating cell growth and survival. J Mol Med 77: 282-293, 1999

6. Bellosillo B, Villamor N, Lopez-Guillermo A, et al: Spontaneous and drug-induced apoptosis is mediated by conformational changes of Bax and Bak in B-cell chronic lymphocytic leukemia. Blood 100: 1810-1816, 2002.

7. Chanan-Khan A and Porter CW: Immunomodulating drugs for chronic lymphocytic leukaemia. Lancet Oncol 7: 480-488, 2006.

8. Chanan-Khan A, Miller KC, Musial L, et al: Clinical efficacy of lenalidomide in patients with relapsed or refractory chronic lymphocytic leukemia: results of a phase II study. J Clin Oncol 24: 5343-5349, 2006

9. Awan FT, Kay NE, Davis ME, et al: Mcl-1 expression predicts progression-free survival in chronic lymphocytic leukemia patients treated with pentostatin, cyclophosphamide and rituximab. Blood 113: 535-537, 2009.

10. Lamanna N, Jurcic JG, Noy A, et al: Sequential therapy with fludarabine, high-dose cyclophosphamide and rituximab in previously untreated patients with chronic lymphocytic leukemia produces high-quality responses: molecular remissions predict for durable complete responses. J Clin Oncol 27: 491-497, 2009.

11. Barton JC: Thalidomide and dexamethasone therapy of myeloma in a patient with previously untreated B-chronic lymphocytic leukemia. Am J Hematol 74: 205-207, 2003.

12. Mauro FR, Foa R, Meloni G, et al: Fludarabine, ara-C, novantrone and dexamethasone (FAND) in previously treated chronic lymphocytic leukemia patients. Haematologica 87: 926-933, 2002.

13. Chanan-Khan A, Miller KC, Takeshita K, et al: Results of a phase 1 clinical trial of thalidomide in combination with fludarabine as initial therapy for patients with treatmentrequiring chronic lymphocytic leukemia (CLL). Blood 106: 3348-3352, 2005 .

14. Franks ME, Macpherson GR and Figg WD: Thalidomide. Lancet 363: 1802-1811, 2004.

15. Rajkumar SV, Blood E, Vesole D, Fonseca R and Greipp PR: Phase III clinical trial of thalidomide plus dexamethasone compared with dexamethasone alone in newly diagnosed multiple myeloma: a clinical trial coordinated by the Eastern Cooperative Oncology Group. J Clin Oncol 24: 431-436, 2006.

16. Campbell MJ, Zelenetz AD, Levy S and Levy R: Use of family specific leader region primers for PCR amplification of the human heavy chain variable region gene repertoire. Mol Immunol 29: 193-203, 1992.

17. Hamblin TJ, Davis Z, Gardiner A, Oscier DG and Stevenson FK: Unmutated $\operatorname{Ig} \mathrm{V}(\mathrm{H})$ genes are associated with a more aggressive form of chronic lymphocytic leukemia. Blood 94: 1848-1854, 1999.

18. Dohner H, Stilgenbauer S, Benner A, et al: Genomic aberrations and survival in chronic lymphocytic leukemia. N Engl J Med 343: 1910-1916, 2000.

19. Best OG, Ibbotson RE, Parker AE, Davis ZA, Orchard JA and Oscier DG: ZAP-70 by flow cytometry: a comparison of different antibodies, anticoagulants and methods of analysis. Cytometry B Clin Cytom 70: 235-241, 2006.

20. Hallek M and Eichhorst BF: Chemotherapy combination treatment regimens with fludarabine in chronic lymphocytic leukemia. Hematol J 5: S20-S30, 2004. 
21. Keating MJ, O'Brien S, Kontoyiannis D, et al: Results of first salvage therapy for patients refractory to a fludarabine regimen in chronic lymphocytic leukemia. Leuk Lymphoma 43: 1755-1762, 2002.

22. Perkins JG, Flynn JM, Howard RS and Byrd JC: Frequency and type of serious infections in fludarabine-refractory B-cell chronic lymphocytic leukemia and small lymphocytic lymphoma: implications for clinical trials in this patient population. Cancer 94: 2033-2039, 2002.

23. Laurenti L, Piccioni P, Tarnani M, et al: Low-dose thalidomide in combination with oral fludarabine and cyclophosphamide is ineffective in heavily pre-treated patients with chronic lymphocytic leukemia. Leuk Res 31: 253-256, 2007.

24. Marriott JB: TNF-alpha antagonists: monoclonal antibodies, soluble receptors, thalidomide and other novel approaches. Expert Opin Investig Drugs 6: 1105-1108, 1997.
25. Podhorecka M, Halicka HD, Klimek P, Kowal M and Dmoszynska A: Thalidomide induces phosphorylation of histone $\mathrm{H} 2 \mathrm{AX}$ and increases rate of apoptosis caused by fludarabine in malignant lymphocytes of chronic lymphocytic leukemia in short-term cell cultures. Leuk Res 33: 997-1000, 2009.

26. Giannopoulos K, Dmoszynska A, Kowal M, et al: Thalidomide exerts distinct molecular antileukemic effects and combined thalidomide/fludarabine therapy is clinically effective in highrisk chronic lymphocytic leukemia. Leukemia 23: 1771-1778, 2009.

27. Pettitt AR, Matutes E and Oscier D: Alemtuzumab in combination with high-dose methylprednisolone is a logical, feasible and highly active therapeutic regimen in chronic lymphocytic leukaemia patients with p53 defects. Leukemia 20: 1441-1445, 2006. 